\title{
Cost-Driven Residential Energy Management for Adaption of Smart Grid and Local Power Generation
}

\author{
Weiliang Zhao ${ }^{\mathrm{a}^{*}}$ \\ Paul Cooper ${ }^{\mathrm{a}}$ \\ Pascal Perez \\ Lan Ding ${ }^{\mathrm{a}}$
}

\begin{abstract}
A smart grid provides dynamic pricing signals which make users be possible to adjust their power demands accordingly. Renewable energy technologies equip a large number of residential homes the capability of local power generation. The varying price of power supply from a smart grid and the existence of local power generation bring opportunities and challenges for energy management at residential homes. This paper proposes a cost-driven residential energy management approach for the adaption of smart grid and local power generation. The target system makes cost-driven scheduling of household appliances by considering the real-time and/or predictable status of smart grid, local power generation, and power consumption demands. The proposed approach minimizes the overall daily electricity cost of household appliances by taking into account both weather and electricity tariff forecasts, predictable home activities, and the flexibility of electricity use.
\end{abstract}

\section{Key words: Smart Grid; Dynamic Power Pricing; Renewable Energy; Power Demand Side Management.}

\section{Introduction}

Smart grids have become a promising means of the integrated management of electricity demand and supply. The smart grid is a convergence of information technology and communication technology for power system engineering by enabling utilities to make more efficient use of their existing assets through demand response, peak shaving, and service quality control $^{1}$. A smart grid normally introduces variable electricity tariffs to reduce peak load across the network and thereby defers the need for investment in network augmentation ${ }^{2}$.

Smart homes utilize modern computer and communication technology in residential buildings to perform a variety of tasks with "ambient intelligence" with features that can benefit users for comfort, convenience, cost-reducing etc. ${ }^{3-4}$. Many residential homes have installed renewable local power generation devices such as solar PV. The existence of local renewable energy

\footnotetext{
${ }^{a}$ Sustainable Building Research Centre, University of Wollongong, NSW 2522, Australia

${ }^{\mathrm{b}}$ SMART Infrastructure Facility, University of Wollongong, NSW 2522, Australia

*Corresponding author: Weiliang Zhao weiliang_zhao@uow.edu.au

http://dx.doi.org/10.14453/isngi2013.proc.53
} 
sources $^{5}$ changes a residential home from a pure power buying party to a buying/selling party when it connects to the smart grid. The introduction of variable electricity tariffs from smart grids and bi-fold buying/selling role of a residential home bring the energy management tasks at residential homes new opportunities and challenges. In order to adapt the dynamic electricity tariffs of smart grids and equipped local power generation capability at residential homes, a costdriven residential energy management approach is proposed. The target smart home system makes cost-driven scheduling by considering the real-time and/or predictable status of smart grid, local power generation, and power consumption demands. The proposed smart home system will support the automation of a residential home for the end-use of electricity from a smart grid with minimum electricity cost.

This paper describes a cost-driven energy management approach for adaption of smart grid and local power generation as an extension of our previous work ${ }^{6}$. The energy models and profiles of electricity facility and household appliances are refined and the genetic algorithm based solution is proposed. The target power smart home system is the central point to manage and control electricity consumption of household appliances and local power generation in the context of smart grid. The approach aims to minimize the cost of a residential home by taking account of home occupants' activity plans, weather conditions, local renewable energy generation, varying price of power supply from a smart grid, and the flexible and inflexible electricity consumptions of household appliances.

This paper is organised as follow. Section 2 describes energy models and profiles of electricity facility and household appliances at residential homes. Section 3 reviews our previous work which formalizes the scheduling of electricity consumption and local generation at residential homes as an optimization problem. Section 4 presents the genetic algorithm based solution of the identified optimization problem. Section 5 provides a conclusion for the paper.

\section{Energy Models and Profiles of Electric Facility and Household Appliances}

Bonino and colleagues have proposed a semantic energy information publishing framework ${ }^{7}$. This framework is lacking of the capability to express the electricity consumption details in the context of weather conditions and occupant activities. This section describes our energy models for the smart grid, local power resources, and individual household appliances, which are the foundation for electrical energy information to be collected, organized, searched, and utilized. The category of electricity facility and household appliances in a residential house is as in Figure 1. 


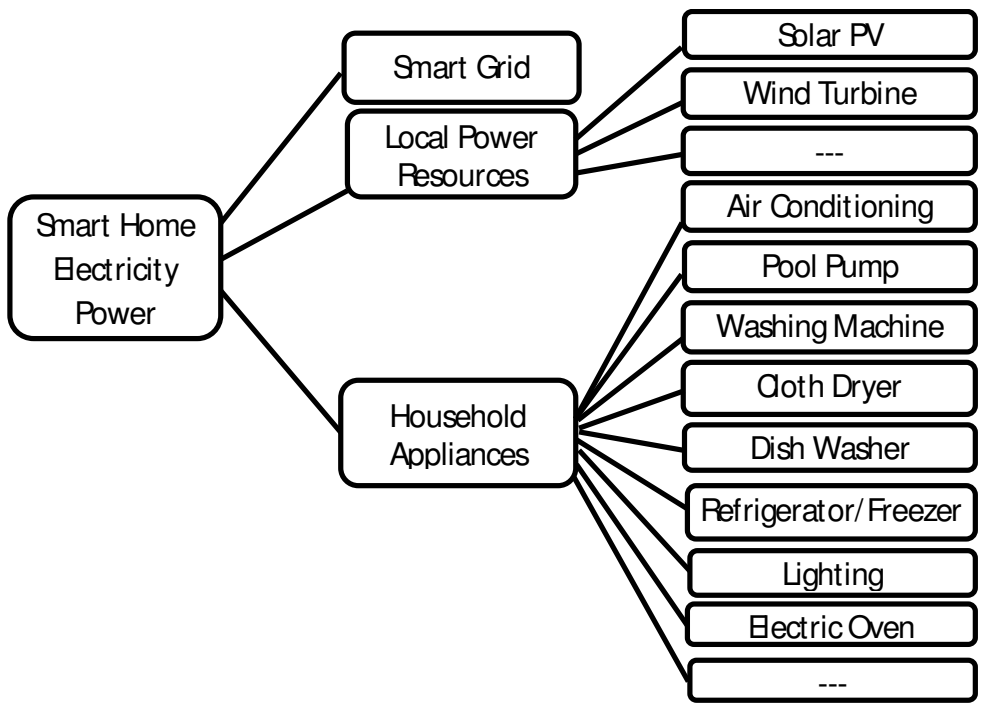

Figure 1. Electric Facility and Household Appliances.

Each element in the above category has its energy model and will be implemented with a specific data structure in database or profile document. The consumption status of an individual device is considered under conditions as day_type, occupants_activities_plan, and weather. The day_type may have values as "week_days", "week_ends", or "public_holidays". The occupants_activity_plan may have values as "week_day", "week_end", "home_holiday", and "home_away_holidays". The weather may include month, temperature, wind, and sunshine_status. The home occupants' activities plan may include energy consumption related activities and people number at home. Due to the paper length limit, we will not provide all details for possible values of these conditions. As an example, the sunshine_status may have the value as "sunny", "mostly_ sunny", "cloudy", or "raining".

Here we provide the generic descriptions of the energy models for electricity facility and household appliances. Most of these data may be collected by the installed ZigBee Panel Meter ${ }^{8}$ with time interval of 15 minutes.

- Smart Grid has data fields "time_point, tariff, current, voltage, power_consumption, day_type, weather".

- Solar PV has data fields "time point, current, voltage, power_generation, weather".

- Air Conditioning has data fields "appliance_id, time_point, on_off, mode, current, voltage, power_consumption, temperature_setpoint, day_type, occupants_activities_plan, weather".

- Swimming Pool Pump has data fields "appliance_id, time_point, on_off, mode, current, voltage, power_consumption".

- Washing Machine has data fields "appliance_id, time_point, on_off, mode, current, voltage, power_consumption".

- Cloth Drier has data fields "appliance_id, time_point, on_off, mode, current, voltage, power_consumption".

- Dishwasher has data fields "appliance_id, time_point, on_off, mode, current, voltage, power_consumption". 
- Refrigerator/freezer has data fields "appliance_id, time_point, on_off, mode, current, voltage, power_consumption, day_type, occupants_activities_plan, weather”.

- Lighting has data fields "appliance_id, time_point, current, voltage, power_consumption, day_type, occupants_activities_plan, weather".

- Electrical Oven has data fields "appliance_id, time_point, on_off, mode, current, voltage, power_consumption, day_type, occupants_activities_plan”.

- Other Devices has data fields "appliance_id, time_point, current, voltage, power_consumption" (day_type, occupants_activities_plan, weather may be included).

Energy profiles will be built up based on the above data structures. These energy profiles will be the foundation of the energy analysis and decision support systems for smart electrical energy management at residential homes. The original data of energy profiles could be obtained and updated with the help of smart meters, home sensors, home activity records, weather station reports, power facility providers etc. Due to the page limit of the paper, here we simply assume they are available and will not provide more details of how to establish and update them.

\section{Cost-driven Demand Optimization in the Context of Smart Grid and local Power Generation}

This section presents an overview of our previous work that formalizes the scheduling of electricity consumption and local generation at residential homes as an optimization problem.

\section{A. Power Price Model}

Smart grids normally have variable electricity tariffs. With local power generation, a residential home will buy/sell power from/to a smart grid. The power price model is crucial in the cost-driven demand optimization in the context of smart grid and local power generation. At residential homes, the supply of electrical power is composed of two parts which will be referred to as local-power and grid-power. The voltage of a smart grid at time $t$ is denoted as $V_{\text {grid }}(t)$. It is assumed that electrical power cannot be sent to the smart grid if $V_{\text {geid }}(t)$ is equal to or higher

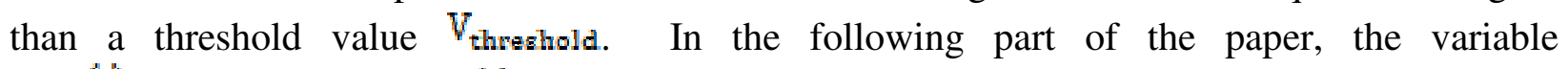
$\mathrm{V}_{\text {gap }}(\mathrm{t})=\mathrm{V}_{\text {threshold }}-\mathrm{V}_{\text {grid }}(\mathrm{t})$ will be used for judging the real time situation. From the point of view of smart grid customers, one of the major features of a smart grid is its time-variable pricing. The local power generation at residential homes must integrate with the smart grid. The power price model is critical in the successful business operation of a smart grid. Considering the characteristics of smart grids, the following price model is adopted:

The grid-power has a variable tariff $T_{\text {grid }}(t)$ which is determined by the smart grid.

- If $V_{g a g}(t)>0$, the selling price of a local power to the smart grid is $T_{\text {locol }}(t)=T_{\text {grid }}(t)-\Delta$. The $\Delta$ is a constant value.

- If $V_{g a p}(t) \leq 0$, a local power cannot be sold to the smart grid.

\section{B. Demand Optimisation}

There are $\mathrm{n}$ household appliances with power consumption rate $\mathrm{U} 1, \mathrm{U} 2, \ldots, \mathrm{Un}$ and $\mathrm{m}$ local power resources with power generation rate $\mathrm{R} 1, \mathrm{R} 2, \ldots, \mathrm{Rm}$. The $\mathrm{Ui}(\mathrm{i}=0, \ldots, \mathrm{n})$ has variables denoted by a vector $\mathbf{x}_{\mathbf{i}}$. These variables reflect the flexibilities of power consumption of 
household appliances. The $\mathrm{Ri}(\mathrm{i}=0, \ldots, \mathrm{m})$ has variables denoted by a vector $y_{i}$. These variables reflect the flexibilities of power generation of local power sources. An example of variables is the starting time of an electric facility. Referred to energy models and profiles for individual electrical entities described previously, $U_{i}\left(x_{i}, t\right)(i=1, \ldots, n)$ and $R_{i}\left(y_{i}, t\right)(i=1, \ldots, m)$ are built up in a specific set of day type, occupants activities plan, and weather forecast.

With $X_{\text {to represent }\left[X_{i}(i=1, m, n)\right.}$ and $Y$ to represent $\left[y_{i}(i=1, \ldots, m)\right]$, the net electrical consumption rate at a specific time point $t$ is

$$
P(\boldsymbol{X}, \boldsymbol{Y}, t)=\sum_{i=1}^{n} U_{i}\left(\boldsymbol{x}_{i}, t\right)-\sum_{i=1}^{m} R_{i}\left(\boldsymbol{y}_{i}, t\right)
$$

Please, note that the above net electrical consumption rate $\mathbf{P}(\mathrm{t})$ could be either positive and negative. The negative value means that the residential home generates more electrical power than what it is consuming at time t. The residential home may buy from or sell to the smart grid electrical power. The net cost rate of the residential home is:

$$
\begin{gathered}
\text { If } P(X, Y, t)==0, C(X, Y, t)=0 \\
\text { if } P(X, Y, t)>0, C(X, Y, t)=P(X, Y, t) \times T_{\text {grid }}(t) \\
\text { if } P(X, Y, t)<0 \\
\text { if } V_{g a g(t)} \leq 0, C(X, Y, t)=0 \\
\text { if } V_{g a p t}>0, C(X, Y, t)=P(X, Y, t) \times T_{\text {Wal }}(t)
\end{gathered}
$$

In the proposed approach for electrical energy management, a specific time period $\mathrm{T}$ is considered in the prediction, recommendation, and schedule of electrical consumption and local generation. A practical period could be a whole day as 24 hours from 8:00 AM to the following day's 8:00 AM. The specific time period $\mathrm{T}$ is divided into $\mathrm{N}$ time intervals. An individual time interval is $\Delta \mathrm{T}=\mathrm{T} / \mathrm{N}$. The total cost of the residential home in time period $\mathrm{T}$ is:

$$
C_{\text {total }}(X, Y)=\sum_{t=0}^{W} C(X, Y, t) \times \Delta \mathrm{T}
$$

The schedules of electrical consumption and local generation at a residential home becomes the optimization problem for minimizing the total cost of electrical power while satisfying power requirements at a residential home.

\section{Genetic Algorithm based Solution of Identified Optimization Problem}

The optimization problem is a typical nonlinear problem that conditional judgments based on dynamic variables are embedded in the cost calculation. In the identified optimization problem, there are multiple variables to be optimized. The method of the whole variable space searching calculates costs for all possible ways and compares them to get the minimum. When the number of variables to be optimized is too big, the whole variable space searching will become impossible. The previous work $^{6}$ has provided an approximate solution of the identified optimization problem based on first principles. The correlations among consumption demands of household appliances have not been considered. It is necessary to find a robust and powerful solution which can cover a wide range of situations in the identified optimization problem.

The genetic algorithm proposed by Holland ${ }^{9}$ provides a robust and efficient optimization solution to cost-driven scheduling. The optimization procedure of a genetic algorithm generally includes four main steps: selection, crossover, mutation and evaluation. Using a biological 
metaphor, the optimization procedure successively uses 'gene' selection, crossover and mutation operations, before performing a recursive fitness evaluation until final convergence.

In this project, we choose Python as the programming language and the Pygene package ${ }^{10}$ to perform the computing tasks. Pygene is a simple and easily understandable library with a rich set of primitive classes for genetic algorithms and genetic programming in python. The development platform is Intel(R) Core ${ }^{\mathrm{TM}}$ i7-2640 CPU @ 2.80GHz 2.80 GHz with $8.00 \mathrm{~GB}$ RAM. The Python version is 2.7.3 under an Integrated Development Environment JetBrains PyCharm 2.6.1.

In the implementation, classes FloatGeneMax, MendelOrganism, and Population in pygene packages are employed as the major working horses. The fitness function implements the total cost of the residential home in a specific scheduling time period which is the whole 24-hour day from 4:00 AM of next day to 4:00 AM of the day after next day. The individual time interval is 15 minutes. The calculation follows the formula in section 3.2. A set of genes represent the variables described in section 3.2 which reflect the flexibilities of power consumption of household appliances and power generation of local power sources. The constraints are that all the power consumption tasks of household appliances and power generation tasks of local power sources must be completed in the scheduling time period. In our implementation of FloatGeneMax, both the crossover parameter and the mutation parameter are 0.5. We have achieved stable computing results for variable numbers from 1 to 10.

As a comparison, we have tried to use the method of whole space searching to solve the optimization of total cost. The computing time of whole space searching will increase exponentially when the number of variables becomes bigger. When the number of variables is bigger than 3, the whole space searching becomes impossible. Comparing with the approximate solution by Zhao et al. ${ }^{6}$, the genetic algorithm provides more accurate optimization results.

\section{Conclusion}

This paper reports our work-in-progress result of proposed approach to minimize the overall daily electricity cost of household appliances by taking into account weather and electricity tariff forecasts, predictable home activities, and the flexibility of electricity use. The energy models and profiles of electric facility and household appliances have been described. The cost-driven scheduling of power consumption and generation has been identified as an optimization problem. A genetic algorithm based solution has been developed to achieve more accurate optimization results comparing with previous work that has not considered the correlations among consumption demands of household appliances.

\section{References}

${ }^{1}$ Farhangi. H., "The path of the smart grid", IEEE Power and Energy Magazine, Vol. 8, No. 1, 2010, pp. 18 -28. http://dx.doi.org/10.1109/MPE.2009.934876

${ }^{2}$ Mohsenian-Rad, A.-H., and Leon-Garcia, A., "Optimal residential load control with price prediction in real-time electricity pricing environment”, IEEE Trans. on Smart Grid, Vol. 1, No. 2, 2010, pp. 120-133. http://dx.doi.org/10.1109/TSG.2010.2055903

${ }^{3}$ Cook, D. J., "How smart is your home?", Science, Vol. 335, No. 8-13, 2012, pp. 1579-1581. 
International Symposium for Next Generation Infrastructure October 1-4, 2013, Wollongong, Australia

${ }^{4}$ Davidoff, S., Lee, M., Yiu, C., Zimmerman, J., and Dey., A., "Principles of smart home control. In Ubiquitous Computing", Lecture Notes in Computer Science, Springer Berlin / Heidelberg, Vol. 4206, 2006, pp. 19-34.

${ }^{5}$ Bert, J. M., Vries, D., Detlef, P., Van, V., and Hoogwijk, M. M., "Renewable Energy Sources: Their global potential for the first-half of the 21st century at a global level: An integrated approach", Energy Policy, Vol. 35, No. 4, 2007, pp. 2590 - 2610. http://dx.doi.org/10.1016/j.enpol.2006.09.002

${ }^{6}$ Zhao, W., Ding, L., Cooper, P., and Perez, P., "Smart Home Electricity Management in the Context of Local Power Resources and Smart Grid", Journal of Clean Energy Technologies, Vol. 2, No. 1, 2014, pp. 73-79. http://dx.doi.org/10.7763/JOCET.2014.V2.95

${ }^{7}$ Bonino, D., Corno, F., and Razzak, F., "Enabling machine understandable exchange of energy consumption information in intelligent domotic environments", Energy and Buildings, Vol. 43, No. 6, 2011, pp. 1392-1402. http://dx.doi.org/10.1016/j.enbuild.2011.01.013

${ }^{8}$ Jetlun, Zigbee panel meter, URL: http://jetlun-estore.myshopify.com/products/zigbee-panelmeter

${ }^{9}$ Holland., J. H., Adaption in Natural and Artificial Systems: An Introductory Analysis with Applications to Biology, Control, and Artificial Intelligence, The University of Michigan Press, 1975.

${ }^{10}$ Pygene, URL: http://freenet.mcnabhosting.com/python/pygene/ 